\title{
Häufiger gramnegative Keime in chronischen Wunden
}

Nach einer Auswertung des Essener Universitätstsklinikums waren im Jahr 2004 mehr als 20\% aller chronischen Wunden mit MRSA kontaminiert. Fünf Jahre später wiederholte man die Untersuchung in der dermatologischen Wundambulanz - die aktuellen Zahlen liegen jetzt vor.

$\mathrm{D}$ ie Auswertung der Wundambulanz umfasste bakteriologische Abstriche von 100 Patienten (60 Frauen, 40 Männer; mittleres Alter 65 Jahre) mit 107 chronischen Beingeschwüren (über drei Monate alte Ulzera). Eine frühere systemische Antibiotika-Behandlung oder eine antiseptische Lokaltherapie waren Ausschlusskriterien. Der Abstrich wurde nach Levine entnommen.

Insgesamt fand man 191 Isolate mit fakultativ pathogenen Bakterien und identifizierte 25 verschiedene Arten. Die Mehrzahl der Wunden war von zwei oder mehr verschiedenen Erregern besiedelt, darunter am häufigsten mit Staphylococcus aureus $(n=59)$, Pseudomonas aeruginosa $(\mathrm{n}=36)$ und Proteus mirabilis ( $\mathrm{n}$ $=17$ ). Bei zehn Patienten fand man MRSA. Bezogen auf die S.-aureus-Isolate waren somit $16,9 \%$ methicillinresistent. Lediglich bei sieben Patienten waren keine Pathogene nachweisbar. Im Vergleich zu den im Jahr 2004 veröffentlichten Daten wurde damit eine markante

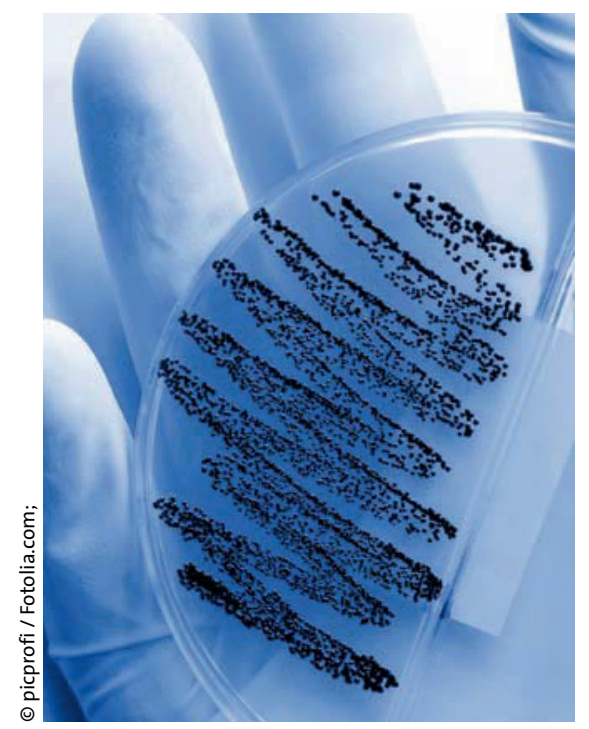

25 verschiedene Arten von Bakterien konnten die Wissenschaftler im Labor nachweisen.
Abnahme des Problemkeims MRSA festgestellt: von 21,5 auf nun $10 \%$ der Fälle - möglicherweise der Effekt einer veränderten Wundversorgung, in der antimikrobielle Maßnahmen wie silberhaltige Verbände und antiseptische Lokaltherapie mit Polihexanid oder Octenidin eine immer größere Rolle spielen, vermuten die Autoren.

An zweiter Stelle der „Hitliste“ stand in der neuen Untersuchung P. aeruginosa, der in immerhin einem Drittel aller Wunden gefunden wurde. Gegenüber den Ergebnissen vor fünf Jahren (24\% der Ulzera) wurde dieser gramnegative Keim deutlich öfter registriert. Eine mögliche Ursache könnte der steigende Gebrauch von Leitungswasser zur Wundspülung sein. (Das Cochrane-Review, das diese Praxis stützt, beruht in der Hauptsache auf Studien aus Ländern, in denen - anders als in Deutschland - Trinkwasser stark gechlort wird.)

Generell scheint die Kolonisation durch gramnegative Bakterien zuzunehmen; z.B. wurde auch P. mirabilis häufiger gefunden. Insgesamt hatte die Anzahl verschiedener in den Wunden nachweisbarer Bakterienarten zugenommen: 25 versus 16 Spezies.

Fazit: Neben dem bereits bekannten Problem mit MRSA sollte den Autoren zufolge die steigende Prävalenz gramnegativer Erreger zukünftig in die Überlegungen zur therapeutischen Strategie mit einbezogen werden. Auch der unkritische Einsatz von Leitungswasser sollte gestoppt werden, sofern keine unmittelbare antiseptische Behandlung folgt. wpa

Körber A et al. Bacterial colonization of chronic leg ulcers: current results compared with data 5 years ago in a specialized dermatology department. J Eur Acad Dermatol Venereol 4. März 2010 [Epub ahead of print]. 